\title{
Editorial
}

\section{To BCVA, or not to BCVA, that is the question}

\author{
Ted Maddess $\mathrm{PhD}^{1}$ and Vincent R Daria $\mathrm{PhD}^{2}$ \\ ${ }^{1}$ Diagnostics for Eye Diseases, ${ }^{2}$ Neurophotonics \\ John Curtin School of Medical Research \\ Australian National University
}

Text: 1278 words

Figure: 1

References: 14

Conflict/competing interest: TM has patents and equity in nuCoria Pty Ltd for a possible objective perimeter. VRD none.

Funding sources: ARC: LP140100763, CE0561903; NHMRC: APP1105944 
Best-corrected visual acuity (BCVA) is has traditionally been the endpoint for clinical trials of interventions for retinal disease. The introduction of optical coherence tomography has meant that structural endpoints, like Central Macular Thickness, have come into use. Are these enough? After all structure is not function, and the central fovea is not the whole retina.

Serious degenerative disease can exist within millimetres of fixation without diminishing acuity. Are less sinister things like minor pigmentary changes or mid-sized drusen near the fovea a threat? If they are causing retinal dysfunction they might be. Not surprisingly the latest NEI/FDA AMD and inherited Retinal Diseases endpoints workshop reaffirmed that functional tests other than BCVA could be used as alternative endpoints in trials. ${ }^{1}$ Microperimeters might be good candidates for those endpoints given their ability to assess visual function both within and around the fovea.

The available microperimeters share features of their progenitor, the MP1 (Nidek, Aichi, Japan). Microperimeters combine a non-mydriatic camera, stabilization of eye movements, and the ability to project perimetric stimuli into the eye. Thus, one can watch the retina live as the stimuli are delivered. In this issue Han et al. have studied the newer Macular Integrity Assessment (MAIA) microperimeter (Centervue S.p.A., Padova, Italy). Most studies of the MAIA have used one of two available test methods: a 68-point test that is similar to the 10-2 program of the Humphrey Field Analyser, or the so-called Standard test that examines 37 points within the central 10 degrees. The 37-point test takes about 6 minutes per eye. ${ }^{2}$

Han et al. pose the highly relevant clinical question: does dilation of the pupils affect the perimetry or eye-tracking results of the MAIA? They recruited 10 patients with $x$-linked choroideremia who had residual central vision, and 20 psuedo-phakic control subjects. This provided stable field defects spanning the 10-2 test pattern, and well-defined optics in the controls. Having reduced these sources of variance the authors then examined the reproducibility of the visual field thresholds, and the gaze tracking, with and without dilation. They found no effects of dilation. Is this result unexpected?

A hint is that the imaging system of the MAIA is an infrared scanning confocal system. As the inventors of the scanning laser ophthalmoscope (SLO) said about these systems the "entrance and exit pupils of a conventional indirect ophthalmoscope are interchanged: in the SLO the laser's high radiance allows us to use only a tiny $(1-\mathrm{mm})$ central portion of the eye's pupil for getting light in. The rest of the pupil (as much as $8 \mathrm{~mm}$ ) can be used to collect light scattered back from the retinal focus of the laser beam". ${ }^{3}$ Thus, the imaging light enters the central pupil as a narrow collimated bundle and so should not affected by dilation. As far the reflected light is concerned the confocal design means that only the integral of the light exiting the pupil matters (for each scanned point of the retina) because that what the detector measures in a confocal system (Fig. 1). The eye tracking is based on the resulting IR image so should not be affected by dilation.

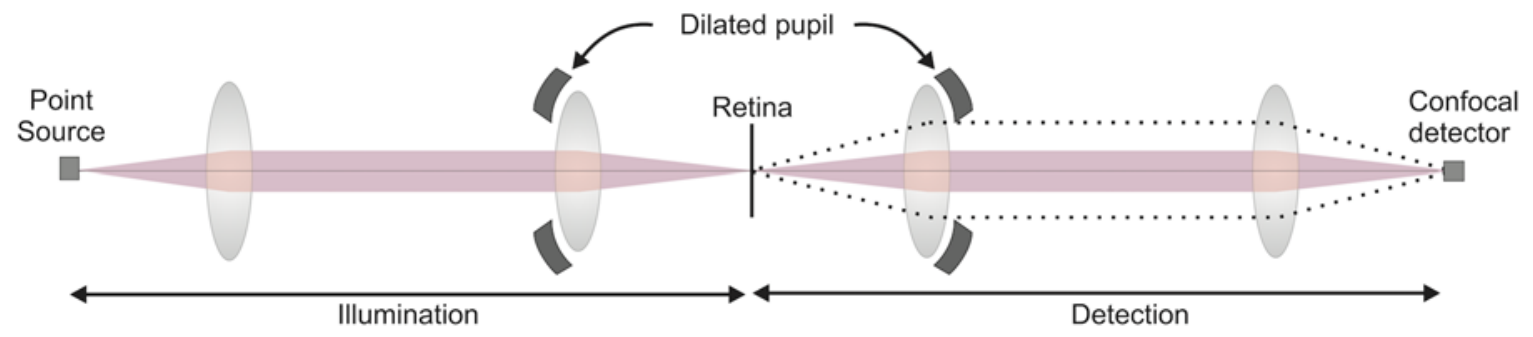

Figure 1. Shows an unfolded representation of the IR imaging engine of the MAIA, separating the illumination and the detection legs of a confocal imaging system. The detection side illustrates the light reflected out of the eye with its similar narrow beam through the central pupil. The dotted line shows the possible (unused) path 
permitted by the dilated pupil, which in principle could degrade the point-spread function (PSF) of the detection leg. The resolution of the system will depend on the size of the confocal detector and the illumination PSF. The perimetric stimuli similarly enter the pupil as a tightly collimated beam passing through the central $2 \mathrm{~mm}$ of the pupil (see text).

That is fine for the IR imaging but what about the white perimetric stimuli? Examining the optical design of the MAIA provided in its primary patent it is clear that the perimetric stimuli are delivered through the pupil as a $2 \mathrm{~mm}$ wide collimated bundle. ${ }^{4}$ The demonstration design also gives the beam width for the IR imaging system as $1 \mathrm{~mm}$ for light entering the eye and $3 \mathrm{~mm}$ for light exiting. This may have been altered slightly in the final MAIA design given that the minimum pupil diameter specified by Centervue is $2.5 \mathrm{~mm}$. All this has been confirmed for us by the company. A further patent indicates that IR dots projected into the eye for the autofocus system of the MAIA also have a small exit pupil. Thus, none of these systems would be expected to be affected by aberrations of the dilated pupil. There is one caveat. As far as the IR imaging is concerned, the MAIA projects a thin horizontal line-beam rather than focal spots into the eye. The thin line-beam is scanned vertically and detected by a linear sensor array to acquire a two-dimensional image of the retina. This means that the system has confocal resolution vertically, but standard resolution horizontally. This design allows the system to have a single, slow, scanning mirror to move the line-beam up and down.

Han et al. also discuss the possible effects of the Stiles-Crawford Effect (SCE). The SCE is caused by small diameter cone outer-segments acting as waveguides, like fibre-optics. ${ }^{5}$ This means that light entering the cones becomes trapped in the outer-segment until most of it is absorbed, thus preserving focus. The cones in the retina have a tight angular acceptance angle. This means that light entering the eye as a narrow collimated pencil will strike the cones at different angles as the pencil is translated parallel to, and away from, the optic axis. The result is that the more off-axis the pencil, the less light gets into the cones, and the dimmer the light appears to the owner of the eye.

The authors mention the SC Effect based on the idea that illumination of the cones via a dilated vs. non-dilated pupil would change the angles at which light arrives at the cones. As we have seen the design of the MAIA means that the paths of all light entering the eye is determined by the small exit pupil of the instrument, and so there is no change in the illumination angles on dilation. Some retinal eccentricity-dependent effects may be of interest however. The SCE is strongest at the fovea, where the cones are narrowest, and declines with decreasing cone density. The resulting variable strength of the SCE within the fovea has been used to map the foveal pit. ${ }^{6}$ Beyond these first few degrees the effect gets about three times weaker than centrally, but then it is quite uniform out to 30 degrees or more. $^{7}$

Overall Han et al. have made a very important contribution in proving what the optics would predict: dilation does not affect the results of the MAIA. For all their sophistication, however, microperimeters are still perimeters with all the problems associated with behavioural testing that takes many minutes per eye. The problems are somewhat mitigated by the eye tracking, although a recent report on the MAIA indicates this does not remove retest variability at the steep edges of scotomas, ${ }^{8}$ as one of us has predicted. ${ }^{9}$ Strictly the tracking system of the MAIA does not remove fixational jitter. Instead, it determines the mean preferred retinal locus (PRL) and presents stimuli centred about that retinal point. It tracks the PRL over the test period and then reports the mean \pm SD of the departure from the estimated centration. ${ }^{10}$ The Nidek MP3 also does this. The MAIA additionally reports the area of the ellipse enclosing the fixational jitter, and rates the fixation error as: "stable, relatively unstable, or unstable". ${ }^{2}$ 
Are there other options? We have recently introduced a non-contact form of objective perimeter that has a macular mode. It tests both eyes concurrently in under 7 minutes using responses of the pupils. Its design makes it tolerant of senescent pupils and the macular test has high diagnostic power in early stage diabetes ${ }^{11}$ and AMD. ${ }^{12}$ The presence of slight hypersensitivities within these fields seem to predict which Wet-AMD patient response to anti-VEGF treatment. ${ }^{13}$ We have recently provided evidence that those signals are relayed to the pupillary system from the extra-striate cortex in both diabetes and AMD. ${ }^{14}$

Overall, we need to do more to manage retinal disease than rely on BCVA.

\section{References}

1. Csaky KG. The NEI/FDA endpoints workshop on AMD and inherited retinal diseases. Personal Communication 2016; http://www.arvo.org/Endpoints2016/.

2. Morales MU, Saker S, Wilde C, et al. Reference Clinical Database for Fixation Stability Metrics in Normal Subjects Measured with the MAIA Microperimeter. Trans/ Vis Sci Technol 2016;5:6.

3. Webb RH, Hughes GW, Delori FC. Confocal scanning laser ophthalmoscope. Appl Opt 1987;26:1492-1499.

4. Griggio P, Turra F. Instrument for eye examination. 2013;US8550626 B2 18 pages.

5. Provis J, Dubis A, Maddess T, Carroll J. Adaptation of the central retina for high acuity vision: Cones, the fovea and the avascular zone. Prog Retin Eye Res 2013;35:63-81.

6. Williams DR. Visual consequences of the foveal pit. Invest Ophthalmol Vis Sci 1980;19:653667.

7. Enoch JM, Hope GM. An analysis of retinal receptor orientation. 3. Results of initial psychophysical tests. Invest Ophthalmol 1972;11:765-782.

8. Wu Z, Jung CJ, Ayton LN, Luu CD, Guymer RH. Test-retest repeatability of microperimetry at the border of deep scotomas. Invest Ophthalmol Vis Sci 2015;56:2606-2611.

9. Maddess T. Modelling the relative influence of fixation and sampling errors on test-retestvariability in perimetry. Graefes Archive Ophthalmol 2014;DOI 10.1007/s00417-014-2751-y.

10. Morales MU, Saker S, Mehta RL, Rubinstein M, Amoaku WM. Preferred retinal locus profile during prolonged fixation attempts. Can J Ophthalmol 2013;48:368-374.

11. Sabeti F, Nolan C, Essex R, Kolic M, James AC, Maddess T. Multifocal pupillography identifies changes in visual sensitivity according to severity of diabetic retinopathy in type 2 diabetes. Invest Ophthalmol Vis Sci 2015;56:4504-4513.

12. Sabeti F, Maddess T, Saikal A, Essex RW, James AC, Carle CF. Multifocal pupillography in early age-related macular degeneration. Opt Vis Sci 2014;91:904-915.

13. Sabeti F, Maddess T, Essex RW, James AC. Multifocal pupillography identifies ranibizumab induced changes in retinal function for exudative age-related macular degeneration. Invest Ophthalmol Vis Sci 2012;53:253-260.

14. Sabeti F, James AC, Carle CF, Essex RW, Bell A, Maddess T. Comparing multifocal pupillographic objective perimetry (mfPOP) and multifocal visual evoked potentials (mfVEP) in retinal diseases. Scientific Reports 2017;Accepted 8/02/2017. 